\title{
ESCs keep their options open
}

Two powerful technologies enabled studies of chromatin that revealed features potentially responsible for the pluripotency of embryonic stem cells (ESCs).

Various studies have suggested that the pattern of epigenetic modification may mark a cell's developmental stage. There are many modifications that modulate chromatin structure; among them are methylation of histone $\mathrm{H} 3$ lysine 4 (H3Lys4) and lysine 27 (H3Lys27), which regulate transcription positively and negatively, respectively.

To study the pattern of histone modification, Bradley Bernstein of Massachusetts General Hospital, Harvard Medical School and the Broad Institute, and his colleagues carried out chromatin immunoprecipitation (ChIP) with antibodies against methylated H3Lys 4 or H3Lys27, and then used tiling arrays to analyze larger regions of the genome than was previously possible. Bernstein explains: "Tiling arrays are unique in that rather than focusing on a particular genomic feature like a gene, exon or even a promoter, [they] cover the entire unique sequence of, 50 for example, a chromosome." Prior work had revealed that modifications generally affected short stretches of DNA, but at some genes encoding developmentally important transcription factors, larger regions of tens of kilobases contained modified histones.

Bernstein and colleagues found that large regions of modified histones appear to be a general feature of loci containing 'developmental' genes (Bernstein et al., 2006). In differentiated cells these regions were either transcriptionally active or silent-methylated at H3Lys4 or H3Lys27, respectively. In ESCs, however, the developmental genes were marked by 'bivalent domains' comprising large regions of H3Lys27 methylation harboring smaller regions of H3Lys4 methylation, modifications that were thought to be mutually exclusive. "We might not have believed this," says Bernstein, but he points out that they used a tiled array that contained $60 \mathrm{Mb}$ of genome and saw this methylation pattern repeatedly. "So that is the way that technology

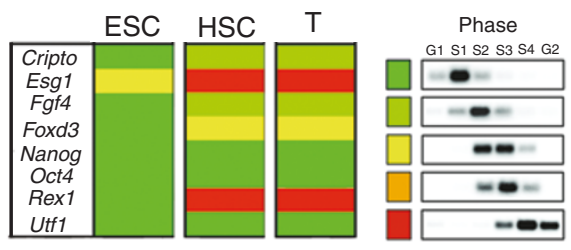

Figure 1 | Replication-timing analysis of ESCS, HSCs and T lymphocytes according to the extent of replication during G1, G2 and throughout S phase. Reprinted with permission from Nature Cell Biology.

helped push the biology, helped us see this unique pattern, and also believe it because we saw it again and again." The authors suggest that these bivalent domains silence genes in ESCs while keeping them poised for selective activation during differentiation.

Addressing similar questions, a team led by Amanda Fisher and Véronique Azuara of the UK Medical Research Council at the Imperial College School of Medicine compared epigenetic profiles of ESCs, hematopoietic stem cells (HSCs) and differentiated Tlymphocytes, hoping to find epigenetic features that distinguish pluripotent cells from those with more restricted developmental potential (Azuara et al., 2006). According to Azuara, they began this study "with the idea that cell identity could be defined by genes being repressed rather than genes being active. So when you are an [ESC], you would keep the potential to express any of the genes, but when you become a lymphocyte, maybe you are identified by the fact that you can't express some genes."

The UK researchers performed replicationtiming analysis, which allows identification of accessible chromatin that is characterized by early replication during $S$ phase. Briefly, they pulse-labeled DNA of cell populations, fractionated the cells according to cell-cycle stage and determined the presence of developmental genes in the labeled DNA by PCR at each stage (Fig. 1). They found that in ESCs many developmentally important genes replicated early but were not expressed; in HSCs and differentiated $\mathrm{T}$ lymphocytes some of these genes were late-replicating, which is characteristic of silenced chromatin. The authors suggest that this is indicative of a difference in transcriptional 'competence' and is a mark of lineage state.

They observed concomitant H3Lys4 and H3Lys27 methylation, and were encouraged that Bernstein et al. also observed this surprising combination at other developmental genes. "So these two papers really complemented each other, and gave you this idea that you might have a significant component of the genome being primed and ready to go in [ESCs] but being held in check by [H3Lys27] methylation," says Fisher. She added that it is likely that somatic stem cells may use a similar mechanism to keep a subset of lineage options available, but not active, and she expects to see papers in the near future describing similar epigenetic patterns in different somatic stem cells.

The exciting findings described in these two articles were possible because of the recent development of microarray tiling technology, which allowed analysis of a large genomic space, and the availability of sophisticated cell sorters to obtain sufficient amounts of cell subpopulations for analysis without the need to arrest cells with drugs. Fisher emphasized that these new technologies have revolutionized this area, and "as more people can get their hands on top-ofthe-range FACS sorting and custom-made arrays, then progress could be much faster." Bernstein echoes the idea that forthcoming technological advances will allow global analyses and adds, "I think as that progresses, we are just going to become exponentially more informed about the epigenetic mechanisms that regulate development and about how aberrant epigenetic regulation can lead to cancer and other diseases."

\section{Irene Kaganman}

\section{RESEARCH PAPERS}

Azuara, V. et al. Chromatin signatures of pluripotent cell lines. Nat. Cell Biol. 8, 532-538 (2006).

Bernstein, B.E. et al. A bivalent chromatin structure marks key developmental genes in embryonic stem cells. Cell 125, 315-326 (2006). 